\title{
SYMMETRIZATION INEQUALITIES FOR PROBABILITY METRIC SPACES WITH CONVEX ISOPERIMETRIC PROFILE
}

\author{
Joaquim Martín* and Walter A. Ortiz ${ }^{\dagger}$ \\ Universitat Autònoma de Barcelona, Department of Mathematics \\ 08193 Bellaterra (Barcelona), Spain; jmartin@mat.uab.cat \\ Universitat Autònoma de Barcelona, Department of Mathematics \\ 08193 Bellaterra (Barcelona), Spain; waortiz@mat.uab.cat
}

\begin{abstract}
We obtain symmetrization inequalities on probability metric spaces with convex isoperimetric profile which incorporate in their formulation the isoperimetric estimator and that can be applied to provide a unified treatment of sharp Sobolev-Poincaré and Nash inequalities.
\end{abstract}

\section{Introduction}

Let $(\Omega, d, \mu)$ be a connected metric space equipped with a separable Borel probability measure $\mu$. The perimeter or Minkowski content of a Borel set $A \subset \Omega$ is defined by

$$
\mu^{+}(A)=\liminf _{h \rightarrow 0} \frac{\mu\left(A_{h}\right)-\mu(A)}{h},
$$

where $A_{h}=\{x \in \Omega: d(x, A)<h\}$ is the open $h$-neighborhood of $A$. The isoperimetric profile $I_{\mu}$ is defined as the pointwise maximal function $I_{\mu}:[0,1] \rightarrow[0, \infty)$ such that

$$
\mu^{+}(A) \geq I_{\mu}(\mu(A))
$$

holds for all Borel sets $A$. An isoperimetric inequality measures the relation between the boundary measure and the measure of a set, by providing a lower bound on $I_{\mu}$ by some function $I:[0,1] \rightarrow[0, \infty)$ which is not identically zero.

The modulus of the gradient of a Lipschitz function $f$ on $\Omega$ (briefly $f \in \operatorname{Lip}(\Omega)$ ) is defined by

$$
|\nabla f(x)|=\limsup _{d(x, y) \rightarrow 0} \frac{|f(x)-f(y)|}{d(x, y)} .
$$

The equivalence between isoperimetric inequalities and Poincaré inequalities was obtained by Maz'ya. Maz'ya's method (see [21], [18] and [8]) shows that given $X=X(\Omega)$ a rearrangement invariant space $^{1}$, the inequality

$$
\left\|f-\int_{\Omega} f d \mu\right\|_{X} \leq c\||\nabla f|\|_{L^{1}}, \quad f \in \operatorname{Lip}(\Omega),
$$

https://doi.org/10.5186/aasfm.2020.4548

2010 Mathematics Subject Classification: Primary 46E35.

Key words: Sobolev-Poincaré inequalities, Nash inequalities, symmetrization, isoperimetric convex profile.

*Partially supported by Grants MTM2016-77635-P, MTM2016-75196-P (MINECO) and 2017SGR358.

${ }^{\dagger}$ Partially supported by Grant MINECO MTM2016-77635-P.

${ }^{1}$ i.e. such that if $f$ and $g$ have the same distribution function then $\|f\|_{X}=\|g\|_{X}$ (see Section 2.2 below). 
holds, if and only if, there exists a constant $c=c(\Omega)>0$ such that for all Borel sets $A \subset \Omega$,

$$
\min \left(\phi_{X}(\mu(A)), \phi_{X}(1-\mu(A))\right) \leq c \mu^{+}(A),
$$

where $\phi_{X}(t)$ is the fundamental function ${ }^{2}$ of $X$ :

$$
\phi_{X}(t)=\left\|\chi_{A}\right\|_{X}, \quad \text { with } \mu(A)=t .
$$

Motivated by this fact, we will say $(\Omega, d, \mu)$ admits a concave isoperimetric estimator if there exists a function $I:[0,1] \rightarrow[0, \infty)$ continuous, concave, increasing on $(0,1 / 2)$, symmetric about the point $1 / 2, I(0)=0$ and $I(t)>0$ on $(0,1)$, such that

$$
I_{\mu}(t) \geq I(t), \quad 0 \leq t \leq 1 .
$$

In their recent work Milman and Martín $^{3}$ (see [17], [19]) proved that $(\Omega, d, \mu$ ) admits a concave isoperimetric estimator $I$, if, and only if, the following symmetrization inequality holds

$$
f_{\mu}^{* *}(t)-f_{\mu}^{*}(t) \leq \frac{t}{I(t)}|\nabla f|_{\mu}^{* *}(t), \quad(f \in \operatorname{Lip}(\Omega))
$$

where $f_{\mu}^{* *}(t)=\frac{1}{t} \int_{0}^{t} f_{\mu}^{*}(s) d s$, and $f_{\mu}^{*}$ is the non increasing rearrangement of $f$ with respect to the measure $\mu$. If we apply a rearrangement invariant function norm $X$ on $\Omega$ (see Sections 2.1 and 2.2 below) to (3) we obtain Sobolev-Poincaré type estimates of the form ${ }^{4}$

$$
\left\|\left(f_{\mu}^{* *}(t)-f_{\mu}^{*}(t)\right) \frac{I(t)}{t}\right\|_{\bar{X}} \leq\left\||\nabla f|_{\mu}^{* *}\right\|_{\bar{X}} .
$$

Example 1. (See [20], [16]) Let $\Omega \subset \mathbf{R}^{n}$ be a Lipschitz domain of measure 1, $X=L^{p}(\Omega), 1 \leq p \leq n$, and $p^{*}$ be the usual Sobolev exponent defined by $\frac{1}{p^{*}}=\frac{1}{p}-\frac{1}{n}$, then $^{5}$

$$
\left\|\left(f^{* *}(t)-f^{*}(t)\right) \frac{I(t)}{t}\right\|_{L^{p}} \simeq\left\|\left(f^{* *}(t)-f^{*}(t)\right)\right\|_{L^{p^{*}, p}},
$$

follows from the fact that the isoperimetric profile is equivalent to $I(t)=c_{n} \min (t, 1-$ $t)^{1-1 / n}$, and Hardy's inequality (here $L^{p^{*}, p}$ is a Lorentz space (see Section 2 below)). In case that we consider $\mathbf{R}^{n}$ with Gaussian measure $\gamma_{n}$, and let $X=L^{p}, 1 \leq p<\infty$, then (compare with [12], [10]), since $I_{\left(\mathbf{R}^{n}, d, \gamma_{n}\right)}(t) \simeq t(\log 1 / t)^{1 / 2}$ for $t$ near zero, we have

$$
\left\|\left(f_{\gamma_{n}}^{* *}(t)-f_{\gamma_{n}}^{*}(t)\right) \frac{I(t)}{t}\right\|_{L^{p}} \simeq\left\|\left(f_{\gamma_{n}}^{* *}(t)-f_{\gamma_{n}}^{*}(t)\right)\right\|_{L^{p}(\log L)^{p / 2}},
$$

where $L^{p}(\log L)^{p / 2}$ is a Lorentz-Zygmund space (see Section 2).

In this fashion in [17], [19], [20] and [16], Milman in collaboration with the first author were able to provide a unified framework to study the classical Sobolev inequalities and logarithmic Sobolev inequalities, moreover embeddings (4) turn out to be the best possible in all the classical cases. However the method used in the proof

\footnotetext{
${ }^{2}$ We can assume with no loss of generality that $\phi_{X}$ is concave.

${ }^{3} \mathrm{~J}$. Martin is grateful to professor Mario Milman for introducing him, around 2005, in the study of the conexion between rearrangements and isoperimetry.

${ }^{4}$ The spaces $\bar{X}$ are defined in Section 2.2 below.

${ }^{5}$ Here the symbol $f \simeq g$ indicates the existence of a universal constant $c>0$ (independent of all parameters involved) such that $(1 / c) f \leq g \leq c f$. Likewise the symbol $f \preceq g$ will mean that there exists a universal constant $c>0$ (independent of all parameters involved) such that $f \leq c g$.
} 
of the previous results cannot be applied with probability measures with heavy tails, since isoperimetric estimators of such measures are non concave. Let us illustrate this phenomenon with some examples (see [7, Propositions 4.3 and 4.4] for Examples 2 and 3 and [22] for Example 4).

Example 2. ( $\alpha$-Cauchy type law) Let $\alpha>0$. Consider the probability measure space $\left(\mathbf{R}^{n}, d, \mu\right)$ where $d$ is the Euclidean distance and $\mu$ is defined by $d \mu(x)=$ $V^{-(n+\alpha)} d x$ with $V: \mathbf{R}^{n} \rightarrow(0, \infty)$ convex. Then there exits $C>0$ such that for any measurable set $A \subset \mathbf{R}^{n}$

$$
\mu^{+}(A) \geq C \min (\mu(A), 1-\mu(A))^{1+1 / \alpha} .
$$

Example 3. (Extended $p$-sub-exponential law) Let $p \in(0,1)$. Consider the probability measure on $\mathbf{R}^{n}$ defined by $d \mu(x)=\left(1 / Z_{p}\right) e^{-V^{p}(x)} d x$ for some positive convex function $V: \mathbf{R}^{n} \rightarrow(0, \infty)$, then there exits $C>0$ such that for any measurable set $A \subset \mathbf{R}^{n}$

$$
\mu^{+}(A) \geq C \min (\mu(A), 1-\mu(A))\left(\log \frac{1}{\min (\mu(A), 1-\mu(A))}\right)^{1-1 / p} .
$$

Example 4. Let $\left(M^{n}, g, \mu\right)$ be a $n$-dimensional weighted Riemannian manifold $(n \geq 2)$ that satisfies the $C D(0, N)$ curvature condition with $N<0$. Then for every Borel set $A \subset\left(M^{n}, g\right)$

$$
\mu^{+}(A) \geq C \min (\mu(A), 1-\mu(A))^{-1 / N} .
$$

Motivated by these examples, we will say $(\Omega, d, \mu)$ admits a convex isoperimetric estimator if there exists a function $I:[0,1] \rightarrow[0, \infty)$ continuous, convex, increasing on $(0,1 / 2)$, symmetric about the point $1 / 2, I(0)=0$ and $I(t)>0$ on $(0,1)$, such that

$$
I_{\mu}(t) \geq I(t), 0 \leq t \leq 1 .
$$

The purpose of this paper is to obtain symmetrization inequalities on probability metric spaces that admit a convex isoperimetric estimator which incorporate in their formulation the isoperimetric estimator and that can be applied to provide a unified treatment of sharp Sobolev-Poincaré and Nash type inequalities. Notice that if $I$ is a convex isoperimetric estimator, then

$$
I(t) \preceq \min (t, 1-t),
$$

therefore (unless $I(t) \simeq \min (t, 1-t))$, the Poincaré inequality

$$
\left\|f-\int_{\Omega} f d \mu\right\|_{L^{1}} \leq c\||\nabla f|\|_{L^{1}}, \quad f \in \operatorname{Lip}(\Omega)
$$

never holds, that means from $|\nabla f| \in L^{1}$ we cannot deduce that $f \in L^{1}$, hence a symmetrization inequality like (3) will not be possible since $f_{\mu}^{* *}$ is defined if, and only if, $f \in L^{1}$.

The paper is organized as follows. In Section 2, we introduce the notation and the standard assumptions used in the paper. This Section also contains the basic background from the theory of rearrangement invariant spaces that we will need. In Section 3 we obtain symmetrization inequalities which incorporate in their formulation the isoperimetric convex estimator. In Section 4 we use the symmetrization inequalities to derive Sobolev-Poincaré and Nash type inequalities. Finally in Section 5 we study in detail Examples 2, 3 and 4. 


\section{Preliminaries}

We recall briefly the basic definitions and conventions we use from the theory of rearrangement-invariant (r.i.) spaces and refer the reader to [2], [13] and [23] for a complete treatment.

We shall consider a connected measure metric spaces $(\Omega, d, \mu)$ equipped with a separable, non-atomic, probability Borel measure $\mu$. Let $\mathcal{M}(\Omega)$ be the set of all extended real-valued measurable functions on $\Omega$. By $\mathcal{M}_{0}(\Omega)$ we denote the class of functions in $\mathcal{M}(\Omega)$ that are finite $\mu$-a.e.

2.1. Rearrangements. For $u \in \mathcal{M}_{0}(\Omega)$, the distribution function ${ }^{6}$ of $u$ is given by

$$
\mu_{u}(t)=\mu\{x \in \Omega: u(x)>t\} \quad(t \in \mathbf{R}) .
$$

The decreasing rearrangement of a function $u$ is the right-continuous non-increasing function from $[0,1)$ into $[0, \infty)$ which is equimeasurable with $u$, i.e.

$$
\mu_{|u|}(t)=\mu\{x \in \Omega:|u(x)|>t\}=m\left\{s \in(0,1): u_{\mu}^{*}(s)>t\right\}, \quad t \in \mathbf{R},
$$

(where $m$ denotes the Lebesgue measure on $(0,1)$ ), and can be defined by the formula

$$
u_{\mu}^{*}(s)=\inf \left\{t \geq 0: \mu_{|u|}(t) \leq s\right\} .
$$

The signed decreasing rearrangement of $f$ is defined by

$$
u_{\mu}^{\star}(t)=\inf \left\{s \in \mathbf{R}: \mu\left\{x \in \Omega: \mu_{u}(x)>s\right\} \leq t\right\},
$$

It follows readily from the definition that

$$
u_{\mu}^{\star}\left(0^{+}\right)=\operatorname{ess} \sup u \quad \text { and } \quad u_{\mu}^{\star}(\infty)=\operatorname{essinf} u .
$$

The maximal function $u_{\mu}^{* *}$ of $u_{\mu}^{*}$ is defined by

$$
u_{\mu}^{* *}(t)=\frac{1}{t} \int_{0}^{t} u_{\mu}^{*}(s) d s=\frac{1}{t} \sup \left\{\int_{E} u(s) d \mu: \mu(E)=t\right\} .
$$

This operation is subadditive, i.e.

$$
(u+v)_{\mu}^{* *}(s) \leq u_{\mu}^{* *}(s)+v_{\mu}^{* *}(s) .
$$

Moreover, since $u_{\mu}^{*}$ is decreasing, $u_{\mu}^{* *}$ is also decreasing and $u_{\mu}^{*} \leq u_{\mu}^{* *}$.

When no confusion ensues, because the measure is clear from the context, or we are dealing with Lebesgue measure, we may simply write $u^{*}$ and $u^{* *}$.

Definition 5. Let $f \in \mathcal{M}_{0}(\Omega)$. We say that $m(f)$ is a median value if

$$
\mu\{f \geq m(f)\} \geq 1 / 2 \text { and } \mu\{f \leq m(f)\} \geq 1 / 2 .
$$

It is easy to see (see for example [17]) that $f_{\mu}^{\star}(1 / 2)$ is a median of $f$. Moreover, if $f$ has 0 median and $f_{\mu}^{\star}$ is continuous then $f_{\mu}^{\star}(1 / 2)=0$.

2.2. Rearrangement invariant spaces. We say that a Banach function space $X=X(\Omega)$ on $(\Omega, d, \mu)$ is rearrangement-invariant (r.i.) space, if

(9) $f \in X$ and $g$ is a $\mu$-measurable such that $f_{\mu}^{*}=g_{\mu}^{*} \Rightarrow g \in X$ and $\|f\|_{X}=\|g\|_{X}$.

If, in the definition of a norm, the triangle inequality is weakened to the requirement that for some constant $C_{X},\|x+y\|_{X} \leq C_{X}\left(\|x\|_{X}+\|y\|_{X}\right)$ holds for all $x$ and $y$, then we have a quasi-norm. A complete quasi-normed space is called a quasi-Banach

\footnotetext{
${ }^{6}$ Note that this notation is somewhat unconventional. In the literature it is common to denote the distribution function of $|u|$ by $\mu_{u}$, while here it is denoted by $\mu_{|u|}$ since we need to distinguish between the rearrangements of $u$ and $|u|$.
} 
space. We will say that $X$ is a quasi Banach rearrangement-invariant (q.r.i.) space if (9) holds.

If $X$ is a r.i. space, since $\mu(\Omega)=1$, for any r.i. space $X(\Omega)$ we have

$$
L^{\infty}(\Omega) \subset X(\Omega) \subset L^{1}(\Omega),
$$

with continuous embeddings.

A r.i. space $X(\Omega)$ can be represented by a r.i. space on the interval $(0,1)$, with Lebesgue measure, $\bar{X}=\bar{X}(0,1)$, such that

$$
\|f\|_{X}=\left\|f_{\mu}^{*}\right\|_{\bar{X}}
$$

for every $f \in X$. A characterization of the norm $\|\cdot\|_{\bar{X}}$ is available (see $[2$, Theorem 4.10 and subsequent remarks]).

A useful property of r.i. spaces states that if

$$
\int_{0}^{r} f_{\mu}^{*}(s) d s \leq \int_{0}^{r} g_{\mu}^{*}(s) d s, \quad \text { holds for all } r>0,
$$

then, for any Banach r.i. space $X=X(\Omega)$,

$$
\|f\|_{X} \leq\|g\|_{X}
$$

Classically conditions on r.i. spaces can be formulated in terms of the boundedness of the Hardy operators defined by

$$
P f(t)=\frac{1}{t} \int_{0}^{t} f(s) d s ; \quad Q f(t)=\int_{t}^{1} f(s) \frac{d s}{s} .
$$

The boundedness of these operators on r.i. spaces can be best described in terms of the so called Boyd indices ${ }^{7}$ defined by

$$
\bar{\alpha}_{X}=\inf _{s>1} \frac{\ln h_{X}(s)}{\ln s} \text { and } \underline{\alpha}_{X}=\sup _{s<1} \frac{\ln h_{X}(s)}{\ln s},
$$

where $h_{X}(s)$ denotes the norm of the compression/dilation operator $E_{s}$ on $\bar{X}$, defined for $s>0$, by

$$
E_{s} f(t)= \begin{cases}f^{*}\left(\frac{t}{s}\right), & 0<t<s \\ 0, & s<t<1\end{cases}
$$

It is well known that

$$
\begin{aligned}
& P \text { is bounded on } \bar{X} \Longleftrightarrow \bar{\alpha}_{X}<1, \\
& Q \text { is bounded on } \bar{X} \Longleftrightarrow \underline{\alpha}_{X}>0 .
\end{aligned}
$$

The next Lemma will be useful in what follows (see [15, Lemma 1]).

Lemma 6. Let $Y$ be a r.i. space on $(0,1)$. Let $\phi_{Y}$ be its fundamental function. Assume that $\phi_{Y}(0)=0$. Then:

(1) If $\bar{\alpha}_{Y}<1$, then for every $\bar{\alpha}_{Y}<\gamma<1$ the function $\phi_{Y}(s) / s^{\gamma}$ is almost decreasing (i.e. $\exists c>0$ s.t. $\phi_{Y}(s) / s^{\gamma} \leq c \phi_{Y}(t) / t^{\gamma}$ whenever $t \leq s$ ).

(2) If $\underline{\alpha}_{Y}>0$, then for every $0<\gamma<\underline{\alpha}_{Y}$ the function $\phi_{Y}(s) / s^{\gamma}$ is almost increasing (i.e. $\exists c>0$ s.t. $\phi_{Y}(s) / s^{\gamma} \leq c \phi_{Y}(t) / t^{\gamma}$ whenever $t \geq s$ ).

(3) If $\underline{\alpha}_{Y}>0$, there exists a concave function $\hat{\phi}_{Y}$ and constant $c>0$ such that

$$
\hat{\phi}_{Y}(t) \simeq \phi_{Y}(t) \quad \text { and } \quad c^{-1} \phi_{Y}(t) / t \leq \frac{\partial}{\partial t} \hat{\phi}_{Y}(t) \leq c \phi_{Y}(t) / t .
$$

\footnotetext{
${ }^{7}$ Introduced by Boyd in [6].
} 
Associated with an r.i. space $X$ there are some useful Lorentz and Marcinkiewicz spaces, namely the Lorentz and Marcinkiewicz spaces defined by the quasi-norms

$$
\|f\|_{M(X)}=\sup _{t} f_{\mu}^{*}(t) \phi_{X}(t), \quad\|f\|_{\Lambda(X)}=\int_{0}^{1} f_{\mu}^{*}(t) \frac{\partial}{\partial t} \phi_{X}(t) .
$$

Notice that

$$
\phi_{M(X)}(t)=\phi_{\Lambda(X)}(t)=\phi_{X}(t)
$$

and that

$$
\Lambda(X) \subset X \subset M(X) .
$$

2.2.1. Examples. Classical Lorentz spaces. The spaces $L^{p, q}(\Omega)$ are defined by the function quasi-norm

$$
\|f\|_{p, q}=\left(\int_{0}^{1}\left(s^{1 / p} f_{\mu}^{*}(s)\right)^{q} \frac{d s}{s}\right)^{1 / q}
$$

when $0<p, q<\infty$, and

$$
\|f\|_{p, \infty}=\sup _{0<t<1} s^{1 / p} f_{\mu}^{*}(s),
$$

when $q=\infty$. Note that $\|f\|_{p, p}=\|f\|_{p}$. (We use the standard convention $\|f\|_{\infty, \infty}=$ $\left.\|f\|_{\infty}\right)$.

Lorentz-Zygmund-spaces. Let $1 \leq p, q \leq \infty$ and $\alpha \in \mathbf{R}$. The spaces $L^{p, q}(\log L)^{\alpha}$ are defined by the function quasi-norm

$$
\|f\|_{p, q, \alpha}=\left(\int_{0}^{1}\left(s^{1 / p}\left(1+\ln \frac{1}{t}\right)^{\alpha} f_{\mu}^{*}(s)\right)^{q} \frac{d s}{s}\right)^{1 / q} .
$$

Weighted q.r.i-spaces. Given $X$ a r.i. space on $\Omega$ and $w$ a weight (i.e a positive measurable function), we define

$$
X(w)=\left\{f:\|f\|_{X(w)}=\left\|f_{\mu}^{*} w\right\|_{\bar{X}}<\infty\right\} .
$$

It is easy to see that $X(w)$ is a q.r.i-space. For example if $X=L^{q}(\Omega)$ and $w(s)=$ $s^{q / p-1}$ then

$$
L^{q}(w)=L^{p, q}(\Omega)
$$

\section{Symmetrization and isoperimetry}

We will assume in what follows that $(\Omega, d, \mu)$ is a connected measure metric spaces equipped with a with a separable, non-atomic, probability Borel measure $\mu$ which admits a convex isoperimetric estimator.

In order to balance generality with power and simplicity, we will assume throughout the paper that our spaces satisfy the following:

Condition 7. We assume that $\Omega$ is such that for every $f \in \operatorname{Lip}(\Omega)$ and every $c \in \mathbf{R}$ we have that $|\nabla f(x)|=0$, a.e. on the set $\{x: f(x)=c\}$.

Theorem 8. Let $I:[0,1] \rightarrow[0, \infty)$ be a convex isoperimetric estimator. The following statements are equivalent:

(1) Isoperimetric inequality: for all Borel sets $A \subset \Omega$,

$$
\mu^{+}(A) \geq I(\mu(A)) .
$$


(2) Ledoux's inequality (cf [14]): for all $f \in \operatorname{Lip}(\Omega)$,

$$
\int_{-\infty}^{\infty} I\left(\mu_{f}(s)\right) \leq \int_{\Omega}|\nabla f(x)| d \mu .
$$

(3) For all function $f \in \operatorname{Lip}(\Omega), f_{\mu}^{\star}$ is locally absolutely continuous, and

$$
\int_{0}^{t}\left(\left(-f_{\mu}^{\star}\right)^{\prime} I(s)\right)^{*} d s \leq \int_{0}^{t}|\nabla f|_{\mu}^{*}(s) d s .
$$

(The second rearrangement on the left hand side is with respect to the Lebesgue measure).

(4) Bobkov's inequality (cf [3]): For all $f \in \operatorname{Lip}(\Omega)$ bounded with $m(f)=0$, and for all $s>0$

$$
\int_{\Omega}|f(x)| d \mu \leq \beta_{1}(s) \int_{\Omega}|\nabla f(x)| d \mu+s \operatorname{Osc}_{\mu}(f),
$$

where $\operatorname{Osc}_{\mu}(f)=\operatorname{ess} \sup f-\operatorname{ess} \inf f$ and $\beta_{1}(s)=\sup _{s<t \leq 1 / 2} \frac{t-s}{I(t)}$.

Proof. (1) $\Rightarrow(2)$ By the co-area inequality applied to $f$ (cf. [4, Lemma 3.1]), and the isoperimetric inequality (14), it follows that

$$
\int_{\Omega}|\nabla f(x)| d \mu \geq \int_{-\infty}^{\infty} \mu^{+}(\{f>s\} ; \Omega) d s \geq \int_{0}^{\infty} I\left(\mu_{f}(s)\right) d s .
$$

$(2) \Rightarrow(3)$ Let $-\infty<t_{1}<t_{2}<\infty$. The smooth truncations of $f$ are defined by

$$
f_{t_{1}}^{t_{2}}(x)= \begin{cases}t_{2}-t_{1} & \text { if } f(x) \geq t_{2} \\ f(x)-t_{1} & \text { if } t_{1}<f(x)<t_{2} \\ 0 & \text { if } f(x) \leq t_{1}\end{cases}
$$

Obviously, $f_{t_{1}}^{t_{2}} \in \operatorname{Lip}(\Omega)$, thus by (14), we get

$$
\int_{-\infty}^{\infty} I\left(\mu_{f_{t_{1}}^{t_{2}}}(s)\right) d s \leq \int_{\Omega}\left|\nabla f_{t_{1}}^{t_{2}}(x)\right| d \mu
$$

By condition 7

$$
\left|\nabla f_{t_{1}}^{t_{2}}\right|=|\nabla f|_{\chi_{\left\{t_{1}<f<t_{2}\right\}}}
$$

and moreover,

$$
\int_{-\infty}^{\infty} I\left(\mu_{f_{t_{1}}^{t_{2}}}(s)\right) d s=\int_{t_{1}}^{t_{2}} I\left(\mu_{f_{t_{1}}^{t_{2}}}(s)\right) d s
$$

Observe that, $t_{1}<z<t_{2}$,

$$
\mu\left\{f \geq t_{2}\right\} \leq \mu_{f_{t_{1}}^{t_{2}}}(z) \leq \mu\left\{f>t_{1}\right\}
$$

Consequently, by the properties of $I$, we have

$$
\int_{t_{1}}^{t_{2}} I\left(\mu_{f_{t_{1}}^{t_{2}}}(z)\right) d z \geq\left(t_{2}-t_{1}\right) \min \left\{I\left(\mu\left\{f \geq t_{2}\right\}\right), I\left(\mu\left\{f>t_{1}\right\}\right)\right\} .
$$

Let us see that $f_{\mu}^{\star}$ is locally absolutely continuous. Indeed, for $s>0$ and $h>0$, pick $t_{1}=f_{\mu}^{\star}(s+h), t_{2}=f_{\mu}^{\star}(s)$, then

$$
s \leq \mu\left\{f(x) \geq f_{\mu}^{\star}(s)\right\} \leq \mu_{f_{t_{1}}^{t_{2}}}(s) \leq \mu\left\{f(x)>f_{\mu}^{\star}(s+h)\right\} \leq s+h .
$$


Combining (18) and (19) we have,

$$
\left(f_{\mu}^{\star}(s)-f_{\mu}^{\star}(s+h)\right) \min \{I(s+h), I(s)\} \leq \int_{\left\{f_{\mu}^{\star}(s)<f<f_{\mu}^{\star}(s+h)\right\}}|\nabla f(x)| d \mu,
$$

which implies that $f_{\mu}^{\star}$ is locally absolutely continuous in $[a, b](0<a<b<1)$. Indeed, for any finite family of non-overlapping intervals $\left\{\left(a_{k}, b_{k}\right)\right\}_{k=1}^{r}$, with $\left(a_{k}, b_{k}\right) \subset$ $[a, b]$, and $\sum_{k=1}^{r}\left(b_{k}-a_{k}\right) \leq \delta$, we have

$$
\mu\left\{\bigcup_{k=1}^{r}\left\{f_{\mu}^{\star}\left(b_{k}\right)<f<f_{\mu}^{\star}\left(a_{k}\right)\right\}\right\}=\sum_{k=1}^{r} \mu\left\{f_{\mu}^{\star}\left(b_{k}\right)<f<f_{\mu}^{\star}\left(a_{k}\right)\right\} \leq \sum_{k=1}^{r}\left(b_{k}-a_{k}\right) \leq \delta .
$$

therefore, combining this fact with (20), we have

$$
\begin{aligned}
\sum_{k=1}^{r}\left(f_{\mu}^{\star}\left(a_{k}\right)-f_{\mu}^{\star}\left(b_{k}\right)\right) \min \{I(a), I(b)\} & \leq \sum_{k=1}^{r}\left(f_{\mu}^{\star}\left(a_{k}\right)-f_{\mu}^{\star}\left(b_{k}\right)\right) \min \left\{I\left(a_{k}\right), I\left(b_{k}\right)\right\} \\
& \leq \sum_{k=1}^{r} \int_{\left\{f_{\mu}^{\star}\left(b_{k}\right)<f<f_{\mu}^{\star}\left(a_{k}\right)\right\}}|\nabla f(x)| d \mu \\
& =\int_{\cup_{k=1}^{r}\left\{f_{\mu}^{\star}\left(b_{k}\right)<f<f_{\mu}^{\star}\left(a_{k}\right)\right\}}|\nabla f(x)| d \mu \\
& \leq \int_{0}^{\sum_{k=1}^{r}\left(b_{k}-a_{k}\right)}|\nabla f|_{\mu}^{*}(t) d t \\
& \leq \int_{0}^{\delta}|\nabla f|_{\mu}^{*}(t) d t,
\end{aligned}
$$

and the local absolute continuity follows.

Now, using (20) we get,

$$
\begin{aligned}
\frac{\left(f_{\mu}^{\star}(s)-f_{\mu}^{\star}(s+h)\right)}{h} \min (I(s+h), I(s)) & \leq \int_{\left\{f_{\mu}^{\star}(s+h)<f<f_{\mu}^{\star}(s)\right\}}|\nabla f(x)| d \mu \\
& \leq \frac{1}{h} \int_{\left\{f_{\mu}^{\star}(s+h)<f \leq f_{\mu}^{\star}(s)\right\}}|\nabla f(x)| d \mu .
\end{aligned}
$$

Letting $h \rightarrow 0$,

$$
\left(-f_{\mu}^{\star}\right)^{\prime}(s) I(s) \leq \frac{\partial}{\partial s} \int_{\left\{f>f_{\mu}^{\star}(s)\right\}}|\nabla f(x)| d \mu .
$$

Let us consider a finite family of intervals $\left(a_{i}, b_{i}\right), i=1, \ldots, m$, with $0<a_{1}<$ $b_{1} \leq a_{2}<b_{2} \leq \cdots \leq a_{m}<b_{m}<1$, then

$$
\begin{aligned}
\int_{\cup_{1 \leq i \leq m}\left(a_{i}, b_{i}\right)}\left(-f_{\mu}^{\star}\right)^{\prime}(s) I(s) d s & \leq \int_{\cup_{1 \leq i \leq m}\left(a_{i}, b_{i}\right)}\left(\frac{\partial}{\partial s} \int_{\left\{|f|>f_{\mu}^{\star}(s)\right\}}|\nabla f(x)| d \mu(x)\right) d s \\
& =\sum_{i=1}^{m} \int_{\left\{f_{\mu}^{\star}\left(b_{i}\right)<|f| \leq f_{\mu}^{\star}\left(a_{i}\right)\right\}}|\nabla f(x)| d \mu(x) \\
& \left.\left.=\sum_{i=1}^{m} \int_{\left\{f_{\mu}^{\star}\left(b_{i}\right)<|f|<f_{\mu}^{\star}\left(a_{i}\right)\right\}}|\nabla f(x)| d \mu(x) \text { (by condition } 7\right)\right)
\end{aligned}
$$




$$
\begin{aligned}
& =\int_{\cup_{1 \leq i \leq m}\left\{f_{\mu}^{\star}\left(b_{i}\right)<|f|<f_{\mu}^{\star}\left(a_{i}\right)\right\}}|\nabla f(x)| d \mu(x) \\
& \leq \int_{0}^{\sum_{i=1}^{m}\left(b_{i}-a_{i}\right)}|\nabla f|_{\mu}^{*}(s) d s .
\end{aligned}
$$

Now by a routine limiting process we can show that for any measurable set $E \subset$ $(0,1)$, with Lebesgue measure equal to $t$, we have

$$
\int_{E}\left(-f_{\mu}^{\star}\right)^{\prime}(s) I(s) d s \leq \int_{0}^{|E|}|\nabla f|_{\mu}^{*}(s) d s
$$

Therefore

$$
\int_{0}^{t}\left(\left(-f_{\mu}^{\star}\right)^{\prime}(\cdot) I(\cdot)\right)^{*}(s) d s \leq \int_{0}^{t}\left(|\nabla f|_{\mu}^{*}(\cdot)\right)^{*}(s) d s
$$

where the second rearrangement is with respect to the Lebesgue measure. Now, since $|\nabla f|_{\mu}^{*}(s)$ is decreasing, we have

$$
\left(|\nabla f|_{\mu}^{*}(\cdot)\right)^{*}(s)=|\nabla f|_{\mu}^{*}(s)
$$

and thus (21) yields

$$
\int_{0}^{t}\left(\left(-f_{\mu}^{\star}\right)^{\prime}(\cdot) I(\cdot)\right)^{*}(s) d s \leq \int_{0}^{t}|\nabla f|_{\mu}^{*}(s) d s .
$$

$(3) \Rightarrow(4)$ Assume first that $f \in \operatorname{Lip}(\Omega)$ is positive, bounded with $m(f)=0$. By 3) we have that $f_{\mu}^{\star}=f_{\mu}^{*}$ (since $f \geq 0$ ) is locally absolutely continuous and $f_{\mu}^{*}(1 / 2)=0($ since $m(f)=0)$. Let $0<s<z \leq 1 / 2$, then

$$
\begin{aligned}
\int_{\Omega}|f(x)| d \mu & =\int_{0}^{1 / 2} f_{\mu}^{*}(z) d z=\int_{0}^{1 / 2} \int_{z}^{1 / 2}\left(-f_{\mu}^{*}\right)^{\prime}(x) d x d z= \\
& =\int_{0}^{1 / 2} z\left(-f_{\mu}^{*}\right)^{\prime}(z) d z-s \int_{0}^{1 / 2}\left(-f_{\mu}^{*}\right)^{\prime}(z) d z+s \int_{0}^{1 / 2}\left(-f_{\mu}^{*}\right)^{\prime}(z) d z \\
& =\int_{0}^{1 / 2} \frac{z-s}{I(z)}\left(-f_{\mu}^{*}\right)^{\prime}(z) I(z) d z+s \int_{0}^{1 / 2}\left(-f_{\mu}^{*}\right)^{\prime}(z) d z \\
& \leq \sup _{s<z \leq 1 / 2} \frac{z-s}{I(z)} \int_{0}^{1 / 2}\left(-f_{\mu}^{*}\right)^{\prime}(z) I(z) d z+s \int_{0}^{1 / 2}\left(-f_{\mu}^{*}\right)^{\prime}(z) d z \\
& \leq \beta_{1}(s) \int_{0}^{1 / 2}\left(-f_{\mu}^{*}\right)^{\prime}(z) I(z) d z+s \int_{0}^{1 / 2}\left(-f_{\mu}^{*}\right)^{\prime}(z) d z
\end{aligned}
$$

Since

$$
s \int_{0}^{1 / 2}\left(-f_{\mu}^{*}\right)^{\prime}(z)=s\left(f_{\mu}^{*}\left(0^{+}\right)-f_{\mu}^{*}(1 / 2)\right) \leq s \operatorname{Osc}_{\mu}(f)
$$


we get

$$
\begin{aligned}
\int_{\Omega}|f(x)| d \mu & \leq \beta_{1}(s) \int_{0}^{t}\left(-f_{\mu}^{*}\right)^{\prime}(z) I(z) d z+s \operatorname{Osc}_{\mu}(f) \\
& \leq \beta_{1}(s) \int_{0}^{1 / 2}\left(\left(-f_{\mu}^{*}\right)^{\prime}(\cdot) I(\cdot)\right)^{*}(t) d t+s \operatorname{Osc}_{\mu}(f) \\
& \leq \beta_{1}(s) \int_{0}^{1 / 2}|\nabla f|_{\mu}^{*}(t) d t+s \operatorname{Osc}_{\mu}(f) \quad(\operatorname{by}(16)) \\
& =\beta_{1}(s) \int_{\Omega}|\nabla f(x)| d \mu+s \operatorname{Osc}_{\mu}(f)
\end{aligned}
$$

In the general case, we follow [3, Lemma 8.3]. Apply the previous argument to $f^{+}=\max (f, 0)$ and $f^{-}=\max (-f, 0)$, which are positive, Lipschitz and have median zero, and we obtain.

$$
\begin{aligned}
& \int_{\{f>0\}}|f(x)| d \mu \leq \beta_{1}(s) \int_{\{f>0\}}|\nabla f(x)| d \mu+s \operatorname{Osc}_{\mu}\left(f^{+}\right), \\
& \int_{\{f<0\}}|f(x)| d \mu \leq \beta_{1}(s) \int_{\{f><0\}}|\nabla f(x)| d \mu+s \operatorname{Osc}_{\mu}\left(f^{-}\right) .
\end{aligned}
$$

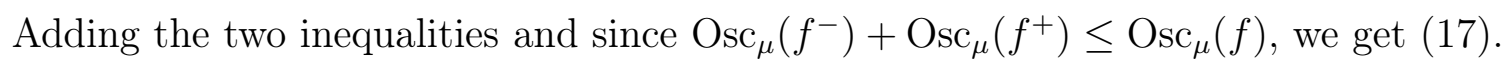

$(4) \Rightarrow(1)$ This part was proved in [3, Lemma 8.3], we include its proof for the sake of completeness. Given a Borel set $A \subset \Omega$ we may approximate the indicator function $\chi_{A}$ by functions with finite Lipschitz seminorm (see [4]) to derive $\mu(A) \leq$ $\beta_{1}(s) \mu^{+}(A)+s$, therefore if $\mu(A)=t$

$$
t-s \leq \beta_{1}(s) \mu^{+}(A),
$$

thus the optimal choice should be

$$
I(t)=\sup _{0<s<t} \frac{t-s}{\beta_{1}(s)} .
$$

\section{Sobolev-Poincaré and Nash type inequalities}

The isoperimetric inequality implies weaker Sobolev-Poincaré and Nash type inequalities, in what follows we will analyze both.

4.1. Sobolev-Poincaré inequalities. The isoperimetric Hardy operator $Q_{I}$ is the operator defined on Lebesgue measurable functions on $(0,1)$ by

$$
Q_{I} f(t)=\int_{t}^{1 / 2} f(s) \frac{d s}{I(s)}, \quad 0<t<1 / 2,
$$

where $I$ is a convex isoperimetric estimator. In this section we consider the possibility of characterizing Sobolev embeddings in terms of the boundedness of $Q_{I}$.

Lemma 9. Let $Y, Z$ be two q.r.i. spaces on $(0,1)$. Assume that there is a constant $C_{0}>0$ such that

$$
\left\|Q_{I} f\right\|_{Y} \leq C_{0}\|f\|_{Z}
$$

Then, there exists a constant $C_{1}>0$ such that

$$
\left\|\bar{Q}_{I} f\right\|_{Y} \leq C_{1}\|f\|_{Z}
$$


where $\bar{Q}_{I}$ is the operator defined on Lebesgue measurable functions on $(0,1)$ by

$$
\bar{Q}_{I} f(t)=\int_{t}^{1 / 2} f(s) \frac{d s}{I(s)}, \quad 0<t<1 .
$$

Proof. Since

$$
\begin{aligned}
\bar{Q}_{I} f(t) & =\chi_{(0,1 / 2)}(t) \bar{Q}_{I} f(t)+\chi_{(1 / 2,1)}(t) \int_{t}^{1 / 2} f(s) \frac{d s}{I(s)} \\
& =\chi_{(0,1 / 2)}(t) Q_{I} f(t)+\chi_{(1 / 2,1)}(t) \int_{t}^{1 / 2} f(s) \frac{d s}{I(s)}
\end{aligned}
$$

it is enough to prove the boundedness of $\chi_{(1 / 2,1)}(t) \int_{t}^{1 / 2} f(s) \frac{d s}{I(s)}$.

For $t \in(1 / 2,1)$, we have that

$$
\begin{aligned}
\int_{t}^{1 / 2} f(s) \frac{d s}{I(s)} & =-\int_{1 / 2}^{t} f(s) \frac{d s}{I(s)}=\int_{1 / 2}^{1-t} f(1-s) \frac{d s}{I(1-s)} \\
& =-\int_{1-t}^{1 / 2} f(1-s) \frac{d s}{I(s)} \quad(\text { since } I(s)=I(1-s)) .
\end{aligned}
$$

Thus

$$
\begin{aligned}
\left\|\chi_{(1 / 2,1)}(t) \int_{t}^{1 / 2} f(s) \frac{d s}{I(s)}\right\|_{Y} & =\left\|\chi_{(1 / 2,1)}(t) \int_{1-t}^{1 / 2} f(1-s) \frac{d s}{I(s)}\right\|_{Y} \\
& =\left\|\chi_{(1 / 2,1)}(1-t) \int_{t}^{1 / 2} f(1-s) \frac{d s}{I(s)}\right\|_{Y}\left(\text { since }\|\cdot\|_{Y} \text { is r.i. }\right) \\
& =\left\|\chi_{(0,1 / 2)}(t) \int_{t}^{1 / 2} f(1-s) \frac{d s}{I(s)}\right\|_{Y} \\
& \leq C\left\|\chi_{(0,1 / 2)}(t) f(1-t)\right\|_{Z} \\
& \leq C\left\|f(t)\left(\chi_{(0,1 / 2)}(1-t)\right)\right\|_{Z} \quad\left(\text { since }\|\cdot\|_{\bar{X}} \text { is r.i. }\right) \\
& \leq C\left\|\chi_{(1 / 2,1)}(t) f(t)\right\|_{Z} \leq C\|f\|_{Z} \cdot
\end{aligned}
$$

Theorem 10. Let $Y$ be a q.r.i. space on $(0,1)$, and let $X$ be a r.i. space on $\Omega$. Assume that there is a constant $C>0$ such that

$$
\left\|Q_{I} f\right\|_{Y} \leq C\|f\|_{\bar{X}}
$$

then, for all $g \in \operatorname{Lip}(\Omega)$ we have that

$$
\inf _{c \in \mathbf{R}}\left\|(g-c)_{\mu}^{*}\right\|_{Y} \preceq\||\nabla g|\|_{X} .
$$

Proof. Given $g \in \operatorname{Lip}(\Omega)$, by part 2 of Theorem $8, g_{\mu}^{\star}$ is locally absolutely continuous on $(0,1)$. Thus, for $t \in(0,1)$, we have that

$$
\begin{aligned}
\left|g_{\mu}^{\star}(t)-g_{\mu}^{\star}(1 / 2)\right| & =\left|\int_{t}^{1 / 2}\left(-g_{\mu}^{\star}\right)^{\prime}(s) d s\right|=\left|\int_{t}^{1 / 2}\left(-g_{\mu}^{\star}\right)^{\prime}(s) I(s) \frac{d s}{I(s)}\right| \\
& =\left|\bar{Q}_{I}\left(\left(-g_{\mu}^{\star}\right)^{\prime}(\cdot) I(\cdot)\right)(t)\right| .
\end{aligned}
$$


Then

$$
\begin{aligned}
\left\|\left|g_{\mu}^{\star}(t)-g_{\mu}^{\star}(1 / 2)\right|\right\|_{Y} & =\left\|\bar{Q}_{I}\left(\left(-g_{\mu}^{\star}\right)^{\prime}(\cdot) I(\cdot)\right)(t)\right\|_{Y} \\
& \preceq\left\|\left(-g_{\mu}^{\star}\right)^{\prime}(\cdot) I(\cdot)\right\|_{\bar{X}} \quad(\text { by }(23) \text { and Lemma 9) } \\
& \preceq\|\nabla g \mid\|_{X} \quad(\text { by }(16)) .
\end{aligned}
$$

Therefore

$$
\begin{aligned}
\inf _{c \in \mathbf{R}}\left\|(g-c)_{\mu}^{*}(t)\right\|_{Y} & =\inf _{c \in \mathbf{R}}\left\|(g-c)_{\mu}^{\star}(t)\right\|_{Y} \leq\left\|\left|g_{\mu}^{\star}(t)-g_{\mu}^{\star}(1 / 2)\right|\right\|_{Y} \\
& \preceq\||\nabla g|\|_{X} .
\end{aligned}
$$

Theorem 11. Let $X$ be a r.i. space on $\Omega$. Assume that $\underline{\alpha}_{X}>0$ or that there is $c>0$ such that the convex isoperimetric estimator $I$, satisfies that

$$
\int_{t}^{1 / 2} \frac{d s}{I(s)} \leq c \frac{t}{I(t)}, \quad 0<t<1 / 2
$$

Then, for all $g \in \operatorname{Lip}(\Omega)$, we have that

$$
\inf _{c \in \mathbf{R}}\left\|(g-c)_{\mu}^{*}(t) \frac{I(t)}{t}\right\|_{\bar{X}} \preceq\||\nabla g|\|_{X} .
$$

Moreover, if $Y$ is a q.r.i. space on $(0,1)$ such that

$$
\left\|Q_{I} f\right\|_{Y} \preceq\|f\|_{\bar{X}}
$$

then for all $\mu$-measurable function $g$ on $\Omega$, we have that

$$
\left\|g_{\mu}^{*}\right\|_{Y} \preceq\left\|g_{\mu}^{*}(t) \frac{I(t)}{t}\right\|_{\bar{X}} .
$$

In particular, for all $g \in \operatorname{Lip}(\Omega)$, we get

$$
\inf _{c \in \mathbf{R}}\left\|(g-c)_{\mu}^{*}\right\|_{Y} \preceq \inf _{c \in \mathbf{R}}\left\|(g-c)_{\mu}^{*}(t) \frac{I(t)}{t}\right\|_{\bar{X}} \preceq\||\nabla g|\|_{X} .
$$

Proof. We associate to the r.i. space $\bar{X}$, the weighted q.r.i. space $Z$ on $(0,1)$ which quasi-norm is defined by

$$
\|f\|_{Z}:=\left\|f^{*}(t) \frac{I(t)}{t}\right\|_{\bar{X}} .
$$

We claim that there is $C>0$ such that

$$
\left\|Q_{I} f\right\|_{Z} \leq C\|f\|_{\bar{X}}
$$

and therefore (25) follows by Theorem 10. 
Case 1. $\underline{\alpha}_{X}>0$ :

$$
\begin{aligned}
\left\|Q_{I} f\right\|_{Z} & =\left\|\frac{I(t)}{t}\left(\int_{t}^{1 / 2} f(s) \frac{d s}{I(s)}\right)^{*}\right\|_{\bar{X}} \leq\left\|\frac{I(t)}{t}\left(\int_{t}^{1 / 2}|f(s)| \frac{d s}{I(s)}\right)^{*}\right\|_{\bar{X}} \\
& =\left\|\frac{I(t)}{t} \int_{t}^{1 / 2}|f(s)| \frac{d s}{I(s)}\right\|_{\bar{X}} \text { (since } Q_{I}|f|(t) \text { is decreasing) } \\
& =\left\|\frac{I(t)}{t} \int_{t}^{1 / 2}|f(s)| \frac{s}{I(s)} \frac{d s}{s}\right\|_{\bar{X}} \\
& \leq\left\|\int_{t}^{1 / 2}|f(s)| \frac{d s}{s}\right\|_{\bar{X}} \quad\left(\text { since } \frac{s}{I(s)} \text { decreases }\right) \\
& \preceq\|f\|_{\bar{X}} \quad\left(\text { since } \underline{\alpha}_{X}>0\right) .
\end{aligned}
$$

Case 2. The convex isoperimetric estimator satisfies (24). Consider $\tilde{Q}_{I}$ defined by

$$
\tilde{Q}_{I} f(t)=\frac{I(t)}{t} Q_{I} f(t) .
$$

We claim that $\tilde{Q}_{I}: L^{1}(0,1) \rightarrow L^{1}(0,1)$ is bounded, and $\tilde{Q}_{I}: L^{\infty}(0,1) \rightarrow L^{\infty}(0,1)$ is bounded, then by interpolation (see [13]) $\tilde{Q}_{I}$ will be bounded on $\bar{X}$. Thus

$$
\begin{aligned}
\left\|Q_{I} f\right\|_{Z} & \leq\left\|Q_{I}|f|\right\|_{Z}=\left\|\frac{I(t)}{t}\left(Q_{I}|f|\right)^{*}(t)\right\|_{\bar{X}} \\
& =\left\|\frac{I(t)}{t} Q_{I}|f|(t)\right\|_{\bar{X}} \quad \text { (since } Q_{I}|f|(t) \text { is decreasing) } \\
& =\left\|\tilde{Q}_{I}|f|(t)\right\|_{\bar{X}} \preceq\|f\|_{\bar{X}}
\end{aligned}
$$

and Theorem 10 applies.

We are going to prove now the claim. By the convexity of $I, \frac{I(t)}{t}$ is increasing for $0<t<1 / 2$, thus

$$
\int_{0}^{s} \frac{I(t)}{t} d t \leq I(s),
$$

therefore

$$
\begin{aligned}
\left\|\tilde{Q}_{I} f\right\|_{1} & \leq \int_{0}^{1} \tilde{Q}_{I}(|f|)(t) d t=\int_{0}^{1 / 2} \frac{I(t)}{t}\left(\int_{t}^{1 / 2}|f(s)| \frac{d s}{I(s)}\right) d t \\
& =\int_{0}^{1 / 2} \frac{|f(s)|}{I(s)}\left(\int_{0}^{s} \frac{I(t)}{t} d t\right) d s \leq \int_{0}^{1 / 2}|f(s)| d s=\|f\|_{1} .
\end{aligned}
$$

Similarly,

$$
\begin{aligned}
\left\|\tilde{Q}_{I} f\right\|_{\infty} & \leq \sup _{0<t<1} \tilde{Q}_{I}(|f|)(t) \leq \sup _{0<t<1 / 2} \frac{I(t)}{t} \int_{t}^{1 / 2}|f(s)| \frac{d s}{I(s)} \\
& \leq\|f\|_{\infty} \sup _{0<t<1 / 2}\left(\frac{I(t)}{t} \int_{t}^{1 / 2} \frac{d s}{I(s)}\right) \leq c\|f\|_{\infty} \text { (by (24)). }
\end{aligned}
$$


To finish the proof of Theorem it remains to see that

$$
\left\|f_{\mu}^{*}\right\|_{\bar{Y}} \preceq\left\|f_{\mu}^{*}(t) \frac{I(t)}{t}\right\|_{\bar{X}} .
$$

Let $C_{\bar{Y}}$ be the constant quasi-norm of $\bar{Y}$, then

$$
\begin{gathered}
\left\|f_{\mu}^{*}\right\|_{\bar{Y}}=\| f_{\mu}^{*}(t) \chi_{(0,1 / 4)}(t)+f_{\mu}^{*}(t) \chi_{(1 / 4,1 / 2)}(t)+f_{\mu}^{*}(t) \chi_{(1 / 2,3 / 4)}(t) \\
+f_{\mu}^{*}(t) \chi_{(3 / 4,1)}(t)\left\|_{\bar{Y}} \leq 4 C_{\bar{Y}}^{2}\right\| f_{\mu}^{*}(t) \chi_{(0,1 / 4)}(t) \|_{\bar{Y}} .
\end{gathered}
$$

Since $f_{\mu}^{*}$ is decreasing,

$$
f_{\mu}^{*}(t) \chi_{(0,1 / 4)}(t) \leq \frac{1}{\ln 2} \int_{t / 2}^{t} f_{\mu}^{*}(s) \frac{d s}{s}=\frac{1}{\ln 2} \int_{t / 2}^{1 / 2} f_{\mu}^{*}(s) \chi_{(0,1 / 4)}(s) \frac{I(s)}{s} \frac{d s}{I(s)} .
$$

Thus

$$
\begin{aligned}
\left\|f_{\mu}^{*}(t) \chi_{(0,1 / 4)}(t)\right\|_{\bar{Y}} & \preceq\left\|Q_{I}\left(f_{\mu}^{*}(\cdot) \chi_{(0,1 / 4)}(\cdot) \frac{I(\cdot)}{\cdot}\right)(t / 2)\right\|_{\bar{Y}} \\
& \preceq\left\|f_{\mu}^{*}(t / 2) \chi_{(0,1 / 4)}(t / 2) \frac{I(t / 2)}{t / 2}\right\|_{\bar{X}}(\text { by }(26)) \\
& \preceq\left\|f_{\mu}^{*}(t) \chi_{(0,1 / 2)}(t) \frac{I(t)}{t}\right\|_{\bar{X}} \preceq\left\|f_{\mu}^{*}(t) \frac{I(t)}{t}\right\|_{\bar{X}} .
\end{aligned}
$$

Combining (28) and (29) we obtain (27).

Remark 12. If $g \in \operatorname{Lip}(\Omega)$ is positive with $m(g)=0$, then it follows from the previous theorem that

$$
\left\|g_{\mu}^{*}(t) \frac{I(t)}{t}\right\|_{\bar{X}} \preceq\||\nabla g|\|_{X},
$$

4.2. Nash inequalities. In this section we obtain Nash type inequalities. We will focus in the following type of probability measures.

Definition 13. Let $\mu$ be a probability measure on $\Omega$, which admits a convex isoperimetric estimator $I$.

(1) Let $\alpha>0$. We will say that $\mu$ is $\alpha$-Cauchy type if

$$
I(t)=c_{\mu} \min (t, 1-t)^{1+1 / \alpha} .
$$

(2) Let $0<p<1$. We will say that $\mu$ is a extended $p$-sub-exponential type if

$$
I(t)=c_{\mu} \min (t, 1-t)\left(\log \frac{1}{\min (t, 1-t)}\right)^{1-1 / p} .
$$

In both cases $c_{\mu}$ denotes a positive constant.

Theorem 14. The following Nash inequalities holds:

(1) Let $\mu$ be $\alpha$-Cauchy type. Let $X$ be a r.i. space on $\Omega$ with $\underline{\alpha}_{X}>0$. Let $1<q \leq \infty$ such that $0 \leq 1 / q<\underline{\alpha}_{X}$. Then for all $f \in \operatorname{Lip}(\Omega)$ positive with $m(f)=0$, we have

$$
\|f\|_{X} \preceq \min _{r>1}\left(r\||\nabla f|\|_{X}+\|f\|_{q, \infty} \phi_{X}\left(r^{-\alpha}\right) r^{\alpha / q}\right) .
$$


(2) Let $\mu$ be extended $p$-sub-exponential type. Let $X$ be a r.i. space on $\Omega$. Let $\beta>0$. Then for all $f \in \operatorname{Lip}(\Omega)$ positive with $m(f)=0$, we have

$$
\|f\|_{X} \preceq\||\nabla f|\|_{X}^{\frac{\beta}{\beta+1}}\|f\|_{X\left(\ln \left(\frac{1}{t}\right)^{\beta\left(\frac{1}{p}-1\right)}\right)}^{\frac{1}{\beta+1}} .
$$

Proof. Part 1. Let $f \in \operatorname{Lip}(\Omega)$ positive with $m(f)=0$ and let $\omega(t)=t^{-1 / \alpha}$ $(0<t<1 / 2)$. Let $r>1$ and let $\beta>0$ that will be chosen later. Then

$$
\begin{aligned}
\|f\|_{X} & =\left\|f_{\mu}^{*}\right\|_{\bar{X}} \leq\left\|f_{\mu}^{*}(t) \frac{\omega(t)}{\omega(t)} \chi_{\{\omega<r)\}}(t)\right\|_{\bar{X}}+\left\|f_{\mu}^{*}(t)\left(\frac{\omega(t)}{\omega(t)}\right)^{\beta} \chi_{\{\omega>r\}}(t)\right\|_{\bar{X}} \\
& \leq r\left\|f_{\mu}^{*}(t) t^{1 / \alpha}\right\|_{\bar{X}}+r^{-\beta}\left\|f_{\mu}^{*}(t) t^{-\beta / \alpha} \chi_{\left(0, r^{-\alpha}\right)}(t)\right\|_{\bar{X}} \\
& =r\left\|f_{\mu}^{*}(t) t^{1 / \alpha}\right\|_{\bar{X}}+r^{-\beta}\left\|t^{1 / q} f_{\mu}^{*}(t) t^{-\beta / \alpha-1 / q} \chi_{\left(0, r^{-\alpha}\right)}(t)\right\|_{\bar{X}} \\
& \leq r\left\|f_{\mu}^{*}(t) t^{1 / \alpha}\right\|_{\bar{X}}+r^{-\beta} \sup _{t>0}\left(t^{1 / q} f_{\mu}^{*}(t)\right)\left\|t^{-\beta / \alpha-1 / q} \chi_{\left(0, r^{-\alpha}\right)}(t)\right\|_{\bar{X}} \\
& \leq r\left\|f_{\mu}^{*}(t) t^{1 / \alpha}\right\|_{\bar{X}}+r^{-\beta}\|f\|_{q, \infty}\left\|t^{-\beta / \alpha-1 / q} \chi_{\left(0, r^{-\alpha}\right)}(t)\right\|_{\Lambda(\bar{X})} \text { (by (13)) } \\
& \preceq r\left\|f_{\mu}^{*}(t) t^{1 / \alpha}\right\|_{\bar{X}}+r^{-\beta}\|f\|_{q, \infty} \int_{0}^{r^{-\alpha}} t^{-\beta / \alpha-1 / q} \frac{\phi_{X}(t)}{t} \text { (by Lemma 6) } \\
& =r\left\|f_{\mu}^{*}(t) t^{1 / \alpha}\right\|_{\bar{X}}+r^{-\beta}\|f\|_{q, \infty} J(r) .
\end{aligned}
$$

Let $0 \leq 1 / q<\gamma<\underline{\alpha}_{X}$, by Lemma 6 ,

$$
\int_{0}^{r^{-\alpha}} t^{-\beta / \alpha-1 / q} \frac{\phi_{X}(t)}{t^{\gamma} t^{1-\gamma}} \preceq \frac{\phi_{X}\left(r^{-\alpha}\right)}{r^{-\alpha \gamma}} \int_{0}^{r^{-\alpha}} t^{-\beta / \alpha-1 / q+\gamma-1} .
$$

At this stage we select $0<\beta<\alpha(\gamma-1 / q)$, then

$$
\int_{0}^{r^{-\alpha}} t^{-\beta / \alpha-1 / q+\gamma-1} \preceq r^{-\alpha(-\beta / \alpha-1 / q+\gamma)},
$$

thus

$$
J(r) \preceq \phi_{X}\left(r^{-\alpha}\right) r^{\beta+\alpha / q} .
$$

Inserting this information in (30) and by Remark 12, we get

$$
\begin{aligned}
\|f\|_{X} & \preceq r\left\|f_{\mu}^{*}(t) t^{1 / \alpha}\right\|_{\bar{X}}+\|f\|_{q, \infty} \phi_{X}\left(r^{-\alpha}\right) r^{\alpha / q} \\
& \preceq r\||\nabla f|\|_{X}+\|f\|_{q, \infty} \phi_{X}\left(r^{-\alpha}\right) r^{\alpha / q} .
\end{aligned}
$$

Part 2. Let $f \in \operatorname{Lip}(\Omega)$ positive with $m(f)=0$ and let $\omega(t)=\left(\ln \frac{1}{t}\right)^{\frac{1}{p}-1}$ $(0<t<1 / 2)$. Let $r>1$ and $\beta>0$.

$$
\begin{aligned}
\|f\|_{X} & =\left\|f_{\mu}^{*}\right\|_{\bar{X}} \leq\left\|f_{\mu}^{*}(t) \frac{\omega(t)}{\omega(t)} \chi_{\{\omega<r)\}}(t)\right\|_{\bar{X}}+\left\|f_{\mu}^{*}(t)\left(\frac{\omega(t)}{\omega(t)}\right)^{\beta} \chi_{\{\omega>r\}}(t)\right\|_{\bar{X}} \\
& \leq r\left\|f_{\mu}^{*}(t)\left(\ln \frac{1}{t}\right)^{1-\frac{1}{p}}\right\|_{\bar{X}}+r^{-\beta}\left\|f_{\mu}^{*}(t)\left(\ln \frac{1}{t}\right)^{\beta\left(\frac{1}{p}-1\right)}\right\|_{\bar{X}} \\
& \preceq r\||\nabla f|\|_{X\left(\log \left(\frac{1}{t}\right)^{\beta\left(\frac{1}{p}-1\right)}\right)}+r^{-\beta}\|f\|_{X} \quad \text { (by Remark 12). }
\end{aligned}
$$

We finish taking the inf for $r>1$. 
Remark 15. Let $X$ be a r.i. space on $\Omega$ with $\underline{\alpha}_{X}>0$. Let $1<q \leq \infty$ such that $0 \leq 1 / q<\underline{\alpha}_{X}$. Then

$$
L^{q, \infty}(\Omega) \subset \Lambda(X) \subset X(\Omega)
$$

Effectively, by Lemma 6

$$
\|f\|_{\Lambda(X)}=\int_{0}^{1} f^{*}(t) \frac{\phi_{X}(t)}{t} d t \leq\|f\|_{q, \infty} \int_{0}^{1} \frac{\phi_{X}(t)}{t^{1+1 / q}} d t .
$$

The last integral is finite since taking $0 \leq 1 / q<\gamma<\underline{\alpha}_{X}$, we get

$$
\int_{0}^{1} \frac{\phi_{X}(t)}{t^{1+1 / q}} d t=\int_{0}^{1} t^{1 / q+\gamma-1} \frac{\phi_{X}(t)}{t^{\gamma}} d t \preceq \int_{0}^{1} t^{1 / q+\gamma-1}<\infty .
$$

\section{Examples and applications}

In this section we will apply the previous work to the probability measures introduced in Examples 2, 3 and 4.

5.1. Cauchy type laws. Consider the probability measure space $\left(\mathbf{R}^{n}, d, \mu\right)$ where $d$ is the Euclidean distance and $\mu$ is the probability measure introduced in Example 2. Such measures have been introduced by Borell [5] (see also [3]). Prototypes of these probability measures are the generalized Cauchy distributions ${ }^{8}$ :

$$
d \mu(x)=\frac{1}{Z}\left(\left(1+|x|^{2}\right)^{1 / 2}\right)^{-(n+\alpha)}, \quad \alpha>0 .
$$

A convex isoperimetric estimator for these measures is (see [7, Proposition 4.3]):

$$
I(t)=\min (t, 1-t)^{1+1 / \alpha} .
$$

Obviously for $0<t<1 / 2$, we have

$$
\int_{t}^{1 / 2} \frac{d s}{s^{1+1 / \alpha}} \preceq \frac{t}{t^{1+1 / \alpha}}
$$

Thus by Theorem 11, given a r.i. space $X$ on $\mathbf{R}^{n}$ we get

$$
\inf _{c \in \mathbf{R}}\left\|(g-c)_{\mu}^{*} \frac{\min (t, 1-t)^{1+1 / \alpha}}{t}\right\|_{\bar{X}} \preceq\||\nabla g|\|_{X}, \quad\left(g \in \operatorname{Lip}\left(\mathbf{R}^{n}\right)\right) .
$$

Proposition 16. Let $1 \leq p<\infty, 1 \leq q \leq \infty$. For all $f \in \operatorname{Lip}\left(\mathbf{R}^{n}\right)$ positive with $m(f)=0$, we get

$$
\|f\|_{\frac{p \alpha}{p+\alpha}, q} \preceq\||\nabla f|\|_{p, q} .
$$

(2) For all $s>p$

$$
\|f\|_{p, q} \preceq\||\nabla f|\|_{p, q}^{\frac{\beta}{\beta+1}}\|f\|_{s, \infty}^{\frac{1}{\beta+1}}
$$

where $\beta=\alpha\left(\frac{1}{p}-\frac{1}{s}\right)$.

\footnotetext{
${ }^{8}$ These measures are Barenblatt solutions of the porous medium equations and appear naturally in weighted porous medium equations, giving the decay rate of this nonlinear semigroup towards the equilibrium measure, see [24] and [9].
} 
Proof. (1) By Theorem 11 we get

$$
\left\|f_{\mu}^{*} t^{\frac{1}{\alpha}}\right\|_{p, q} \preceq\||\nabla f|\|_{p, q} .
$$

Now by $[13$, page 76$]$ we have that

$$
\left\|f_{\mu}^{*} t^{\frac{1}{\alpha}}\right\|_{p, q}^{q}=\int_{0}^{1}\left[\left(t^{\frac{1}{\alpha}} f_{\mu}^{*}(t)\right)^{*} t^{\frac{1}{p}}\right]^{q} \frac{d t}{t} \simeq \int_{0}^{1}\left(t^{\frac{1}{\alpha}+\frac{1}{p}} f_{\mu}^{*}(t)\right)^{q} \frac{d t}{t}=\|f\|_{\frac{p \alpha}{p+\alpha}, q}^{q} .
$$

(2) is a direct application of Theorem 14 .

Remark 17. If in the previous Proposition we take $p=q=1$, we obtain

$$
\|f\|_{\frac{\alpha}{\alpha+1}, 1} \preceq\||\nabla f|\|_{1} .
$$

If $\frac{1}{q}=\frac{1}{p}+\frac{1}{\alpha}$, then we get

$$
\|f\|_{p, \frac{p(1+\alpha)}{\alpha}} \preceq\||\nabla f|\|_{q, \frac{p(1+\alpha)}{\alpha}} .
$$

For $p \geq 1$ and $s=\infty$, we have that

$$
\|f\|_{p} \preceq\||\nabla f|\|_{p}^{\frac{\beta}{\beta+1}}\|f\|_{\infty}^{\frac{1}{\beta+1}} .
$$

Inequalities 31 and 32 were proved in [22, Proposition 5.13]. Inequality 33 was obtained in [22, Proposition 5.15].

We close this section with the following optimality result:

Theorem 18. Let $\alpha>0$. Let $\bar{X}$ be a r.i. space on $(0,1)$ and let $Z$ be a q.r.i. space on $(0,1)$. Assume that for any probability measure $\mu$ be of $\alpha$-Cauchy type in $\mathbf{R}^{n}$, there is a $C_{\mu}>0$, such that for all $f \in \operatorname{Lip}\left(\mathbf{R}^{n}\right)$ positive with $m(f)=0$, we get

$$
\left\|f_{\mu}^{*}\right\|_{Z} \leq C_{\mu}\left\||\nabla f|_{\mu}^{*}\right\|_{\bar{X}}
$$

Then for all $g \in \operatorname{Lip}\left(\mathbf{R}^{n}\right)$

$$
\left\|g_{\mu}^{*}\right\|_{Z} \preceq\left\|g_{\mu}^{*}(t) \frac{I(t)}{t}\right\|_{\bar{X}} .
$$

Proof. Let $\mu$ be the Cauchy probability measure on $\mathbf{R}$ defined by

$$
d \mu(s)=\frac{\alpha}{2\left(1+|s|^{2}\right)^{\frac{1+\alpha}{2}}} d s=\varphi(s) d x, \quad s \in \mathbf{R} .
$$

It is known (see [7, Proposition 5.27] and [11]) that its isoperimetric profile is given by

$$
I_{\mu}(t)=\varphi\left(H^{-1}(t)\right)=\alpha 2^{1 / \alpha} \min (t, 1-t)^{1+1 / \alpha}, \quad t \in[0,1],
$$

where $H$ is the distribution function of $\mu$, i.e. $H: \mathbf{R} \rightarrow(0,1)$ is the increasing function given by

$$
H(r)=\int_{-\infty}^{r} \varphi(t) d t
$$

Consider on $\mathbf{R}^{n}$ the product measure $\mu^{n}$, by Proposition 5.27 of [7] the function

$$
I(t)=\frac{c_{\alpha}}{n^{1 / \alpha}} \min (t, 1-t)^{1+1 / \alpha}
$$

is a convex isoperimetric estimator of $\mu^{n}\left(c_{\alpha}\right.$ denotes a positive constant depending only of $\alpha$ ). 
Given a positive measurable function $f$ with supp $f \subset(0,1 / 2)$, consider

$$
F(t)=\int_{t}^{1} f(s) \frac{d s}{I_{\mu}(s)}, \quad t \in(0,1)
$$

and define

$$
u(x)=F\left(H\left(x_{1}\right)\right), \quad x \in \mathbf{R}^{n} .
$$

Then,

$$
|\nabla u(x)|=\left|\frac{\partial}{\partial x_{1}} u(x)\right|=\left|-f\left(H\left(x_{1}\right)\right) \frac{H^{\prime}\left(x_{1}\right)}{I_{\mu}\left(H\left(x_{1}\right)\right)}\right|=f\left(H\left(x_{1}\right)\right) .
$$

Let $A$ be a Young's function and let $s=H\left(x_{1}\right)$. Then,

$$
\int_{\mathbf{R}^{n}} A\left(f\left(H\left(x_{1}\right)\right)\right) d \mu^{n}(x)=\int_{\mathbf{R}} A\left(f\left(H\left(x_{1}\right)\right)\right) d \mu\left(x_{1}\right)=\int_{0}^{1} A(f(s)) d s .
$$

Therefore, by [2, Exercise 5, page 88]

$$
|\nabla u|_{\mu^{n}}^{*}(t)=f^{*}(t)
$$

Similarly

$$
u_{\mu^{n}}^{*}(t)=\int_{t}^{1} f(s) \frac{d s}{I_{\mu}(s)} .
$$

Since $m(u)=0$, by the hypothesis we get

$$
\left\|\int_{t}^{1} f(s) \frac{d s}{I_{\mu}(s)}\right\|_{Z}=\left\|u_{\mu^{n}}^{*}\right\|_{Z} \leq C_{\mu^{n}}\left\||\nabla f|_{\mu^{n}}^{*}\right\|_{\bar{X}}=C_{\mu^{n}}\left\|f^{*}(t)\right\|_{\bar{X}}=C_{\mu^{n}}\|f\|_{\bar{X}} .
$$

Finally, from

we have that

$$
I_{\mu}(t)=\frac{\alpha 2^{1 / \alpha} n^{1 / \alpha}}{c_{\alpha}} I(t)
$$

$$
\left\|Q_{I} f\right\|_{Z} \leq \frac{c_{\alpha} C_{\mu^{n}}}{\alpha 2^{1 / \alpha} n^{1 / \alpha}}\|f\|_{\bar{X}}
$$

and the results follows by Theorem 11 .

5.2. Extended $\boldsymbol{p}$-sub-exponential law. Consider the probability measure on $\mathbf{R}^{n}$ defined by

$$
d \mu(x)=\frac{1}{Z_{p}} e^{-V(x)^{p}} d x=\varphi(x) d x
$$

for some positive convex function $V: \mathbf{R}^{n} \rightarrow(0, \infty)$ and $p \in(0,1)$.

A typical example is $V(x)=|x|^{p}$, and $0<p<1$, which yields to sub-exponential type law.

A convex isoperimetric estimator for these type of measures is (see [7, Proposition 4.5] and [11]):

$$
I(t)=c_{p} \min (t, 1-t)\left(\log \frac{1}{\min (t, 1-t)}\right)^{1-1 / p} .
$$

By Theorem 11, given a r.i. space $X$ on $\mathbf{R}^{n}$ with $\underline{\alpha}_{X}>0$, we get

$$
\inf _{c \in \mathbf{R}}\left\|(g-c)_{\mu}^{*} \frac{c_{p} \min (t, 1-t)\left(\log \frac{1}{\min (t, 1-t)}\right)^{1-1 / p}}{t}\right\|_{\bar{X}} \preceq\||\nabla g|\|_{X}, \quad\left(g \in \operatorname{Lip}\left(\mathbf{R}^{n}\right)\right) .
$$

In the particular case that $X=L^{r, q}$ we obtain 
Proposition 19. Let $1 \leq r<\infty, 1 \leq q<\infty$. For all $f \in \operatorname{Lip}\left(\mathbf{R}^{n}\right)$ positive with $m(f)=0$, we get

(1)

(2) For all $\beta>0$

$$
\|f\|_{L^{r, q}(\log L)^{1-1 / p}} \preceq\||\nabla f|\|_{r, q} .
$$

$$
\|f\|_{r, q} \preceq\||\nabla f|\|_{r, q}^{\frac{\beta}{\beta+1}}\|f\|_{L^{r, q}(\log L)^{\beta(1-1 / p)}}^{\frac{1}{\beta+1}}
$$

Theorem 20. Let $p \in(0,1)$. Let $\bar{X}$ be a r.i. space on $(0,1)$ and let $Z$ be a q.r.i. space on $(0,1)$. Assume that for any extended $p$-sub-exponential law $\mu$ in $\mathbf{R}^{n}$, there is a $C_{\mu}>0$, such that for all $f \in \operatorname{Lip}\left(\mathbf{R}^{n}\right)$ positive with $m(f)=0$, we get

$$
\left\|f_{\mu}^{*}\right\|_{Z} \leq C_{\mu}\left\||\nabla f|_{\mu}^{*}\right\|_{\bar{X}}
$$

Then, for all $g \in \operatorname{Lip}\left(\mathbf{R}^{n}\right)$, we get

$$
\left\|g_{\mu}^{*}\right\|_{Z} \preceq\left\|g_{\mu}^{*}(t) \frac{I(t)}{t}\right\|_{\bar{X}}
$$

Proof. Let $\mu$ be the probability measure on $\mathbf{R}$ with density

$$
d \mu(s)=\frac{e^{-|s|^{p}}}{Z_{p}} d s=\varphi(s) d s, \quad s \in \mathbf{R} .
$$

Its isoperimetric profile is (see [7, Proposition 5.25])

$$
I_{\mu}(t)=\varphi\left(H^{-1}(t)\right)=c_{p} \min (t, 1-t)\left(\log \frac{1}{\min (t, 1-t)}\right)^{1-1 / p}, \quad t \in[0,1],
$$

where $H$ is the distribution function of $\mu$, i.e. $H: \mathbf{R} \rightarrow(0,1)$ is defined by

$$
H(r)=\int_{-\infty}^{r} \varphi(t) d t
$$

Consider on $\mathbf{R}^{n}$ the product measure $\mu^{n}$, by Proposition 5.25 of [7], there exists a positive constant $c$ such that the function

$$
I(t)=c \min (t, 1-t)\left(\log \frac{n}{\min (t, 1-t)}\right)^{1-1 / p}
$$

is a convex isoperimetric estimator of $\mu^{n}$

Let $f$ be a positive measurable function $f$ with $\operatorname{supp} f \subset(0,1 / 2)$, consider

$$
F(t)=\int_{t}^{1} f(s) \frac{d s}{I_{\mu}(s)}, \quad t \in(0,1)
$$

and define

$$
u(x)=F\left(H\left(x_{1}\right)\right), \quad x \in \mathbf{R}^{n} .
$$

Using the same method that in Theorem 18, we obtain

$$
|\nabla u|_{\mu^{n}}^{*}(t)=f^{*}(t) \quad \text { and } \quad u_{\mu^{n}}^{*}(t)=\int_{t}^{1} f(s) \frac{d s}{I_{\mu}(s)} .
$$

Since $m(u)=0$, by the hypothesis we get

$$
\left\|\int_{t}^{1} f(s) \frac{d s}{I_{\mu}(s)}\right\|_{Z}=\left\|u_{\mu^{n}}^{*}\right\|_{Z} \leq C_{\mu^{n}}\left\||\nabla f|_{\mu^{n}}^{*}\right\|_{\bar{X}}=C_{\mu^{n}}\left\|f^{*}(t)\right\|_{\bar{X}}=C_{\mu^{n}}\|f\|_{\bar{X}} .
$$


Finally, from

$$
I_{\mu}(t) \simeq I(t)
$$

we have that

and Theorem 11 applies.

$$
\left\|Q_{I} f\right\|_{Z} \preceq\|f\|_{\bar{X}}
$$

5.3. Weighted Riemannian manifold with negative dimension. Let $\left(M^{n}, g, \mu\right)$ be a $n$-dimensional weighted Riemannian manifold $(n \geq 2)$ that satisfies the $C D(0, N)$ curvature condition with $N<0$. (See [22, Secction 5.4]).

A convex isoperimetric estimator is given by

$$
I(t)=\min (t, 1-t)^{-1 / N} .
$$

Obviously for $0<t<1 / 2$, we have

$$
\int_{t}^{1 / 2} \frac{d s}{s^{-1 / N}} \preceq \frac{t}{t^{-1 / N}}
$$

Thus by Theorem 11, given a r.i. space $X$ on $\mathbf{R}^{n}$ we get

$$
\inf _{c \in \mathbf{R}}\left\|(g-c)_{\mu}^{*} \frac{\min (t, 1-t)^{-1 / N}}{t}\right\|_{\bar{X}} \preceq\|\mid \nabla g\|_{X}, \quad\left(g \in \operatorname{Lip}\left(\mathbf{R}^{n}\right)\right) .
$$

In particular, if $1 \leq p<\infty, 1 \leq q \leq \infty)$ and $X=L^{p, q}$, then for all $f \in \operatorname{Lip}\left(\mathbf{R}^{n}\right)$ positive with $m(f)=0,(1 \leq p<\infty, 1 \leq q \leq \infty)$

$$
\|f\|_{\gamma, q} \preceq\||\nabla f|\|_{p, q},
$$

where $\gamma=\frac{N p}{N-p(N+1)}$ for any $p, q$ satisfying $\frac{N}{N-1} \leq p \leq-N$ and $\frac{1}{q}=\frac{1}{p}-\frac{1}{N}-1$.

And by Theorem 14, we have that

$$
\|f\|_{p, q} \preceq\||\nabla f|\|_{p, q}^{\frac{\beta}{\beta+1}}\|f\|_{s, \infty}^{\frac{1}{\beta+1}}
$$

where $s>p$ and $\beta=\alpha\left(\frac{1}{p}-\frac{1}{s}\right)$.

\section{References}

[1] Barthe, F.: Levels of concentration between exponential and Gaussian. - Ann. Fac. Sci. Toulouse Math. 6, 2001, 393-404.

[2] Bennett, C., and R. Sharpley: Interpolation of operators. - Academic Press, Boston, 1988.

[3] Bobkov, S. G.: Large deviations and isoperimetry over convex probability measures with heavy tails. - Electron. J. Probab. 12, 2007, 1072-1100.

[4] Bobkov, S. G., and C. Houdré: Some connections between isoperimetric and Sobolev-type inequalities. - Mem. Amer. Math. Soc. 129:616, 1997.

[5] Borell, C.: Convex set functions in d-space. - Period. Math. Hungar. 6, 1975, 111-136.

[6] Boyd, D. W.: Indices of function spaces and their relationship to interpolation. - Canad. J. Math. 21, 1969, 1245-1254.

[7] Cattiaux, P., N. Gozlan, A. Gulllin, and C. Roberto: Functional inequalities for heavy tailed distributions and application to isoperimetry. - Electron. J. Probab. 15, 2010, 346-385.

[8] Cerdà, J., J. Martín, and P. Silvestre: Conductor Sobolev-type estimates and isocapacitary inequalities. - Indiana Univ. Math. J. 61, 2012, 1925-1947.

[9] Dolbeault, J., I. Gentil, A. Guillin, and F. Y. Wang: $l^{q}$ functional inequalities and weighted porous media equations. - Potential Anal. 28, 2008, 35-59.

[10] Feissner, G. F.: Hypercontractive semigroups and Sobolev's inequality. - Trans. Amer. Math. Soc. 210, 1975, 51-62. 
[11] Feo, F., M. R. Posteraro, and C. Roberto: Quantitative isoperimetric inequalities for log-convex probability measures on the line. - J. Math. Anal. Appl. 420, 2014, 879-907.

[12] Gross, L.: Logarithmic Sobolev inequalities. - Amer. J. Math. 97, 1975, 1061-1083.

[13] Krein, S. G., Yu. I. Petunin, and E. M. Semenov: Interpolation of linear operators. Transl. Math. Monogr. 54, Amer. Math. Soc., Providence, 1982.

[14] Ledoux, M.: Isopérimétrie et inégalitées de Sobolev logarithmiques gaussiennes. - C. R. Acad. Sci. Paris 306, 1988, 79-92.

[15] Martin, J.: Symmetrization inequalities in the fractional case and Besov embeddings. - J. Math. Anal. Appl. 344, 2008, 99-123.

[16] Martín, J., and M. Milman: Isoperimetry and Symmetrization for Logarithmic Sobolev inequalities. - J. Funct. Anal. 256, 2009, 149-178.

[17] Martin, J., and M. Milman: Pointwise symmetrization inequalities for Sobolev functions and applications. - Adv. Math. 225, 2010, 121-199.

[18] Martín, J., and M. Milman: Sobolev inequalities, rearrangements, isoperimetry and interpolation spaces, concentration, functional inequalities and isoperimetry. - Contemp. Math. 545, Amer. Math. Soc., Providence, RI, 2011, 167-193.

[19] Martin, J., and M. Milman: Fractional Sobolev inequalities: symmetrization, isoperimetry and interpolation. - Astérisque 366, 2014.

[20] Martín, J., M. Milman, and E. Pustylnik: Sobolev inequalities: Symmetrization and self-improvement via truncation. - J. Funct. Anal. 252, 2007, 677-695.

[21] Maz'YA, V. G.: Sobolev spaces. - Springer-Verlag, New York, 1985.

[22] Milman, E.: Beyond traditional curvature-dimension I: new model spaces for isoperimetric and concentration inequalities in negative dimension. - Trans. Amer. Math. Soc. 369, 2017, 3605-3637.

[23] Rakotoson, J. M.: Réarrangement relatif. Un instrument d'estimations dans les problèmes aux limites. - Math. Appl. (Berlin) 64, Springer, Berlin, 2008.

[24] VÁzquez, J. L.: An introduction to the mathematical theory of the porous medium equation. - In: Shape optimization and free boundaries (Montreal, PQ, 1990), NATO Adv. Sci. Inst. Ser. C Math. Phys. Sci. 380, Kluwer Acad. Publ., Dordrecht, 1992, 347-389.

Received 11 June $2019 \bullet$ Accepted 1 October 2019 